\title{
Knockdown of TOR signaling pathway regulator suppresses cell migration and invasion in non-small cell lung cancer via the regulation of epithelial-to-mesenchymal transition
}

\author{
XIANG XU $^{1 *}$, HUANGKAI ZHU ${ }^{1,2^{*}}$, MINGLEI YANG ${ }^{1}$, ENKUO ZHENG $^{1}$, YINJIE ZHOU ${ }^{1}$, \\ JUNJUN NI $^{1}$, RUI LI ${ }^{1}$, ZHENHUA YANG ${ }^{1}$, TI HE ${ }^{3}$ and GUOFANG ZHAO ${ }^{1 *}$ \\ ${ }^{1}$ Department of Cardiothoracic Surgery, Hwa Mei Hospital, University of Chinese Academy of Sciences, \\ Ningbo, Zhejiang 315010; ${ }^{2}$ Zhejiang Provincial Key Laboratory of Pathophysiology, Ningbo, Zhejiang 315000; \\ ${ }^{3}$ Shanghai Genechem Translational Medicine Institute, Shanghai 200120, P.R. China
}

Received January 15, 2019; Accepted October 22, 2019

DOI: $10.3892 /$ etm.2019.8358

\begin{abstract}
Non-small cell lung cancer (NSCLC) is one of the most common cancer types worldwide. Previous studies have indicated that TOR signaling pathway regulator (TIPRL) is involved in the progression of NSCLC. However, the underlying mechanisms of the role of TIPRL in regulating NSCLC metastasis have remained largely elusive. In the present study, the expression pattern of TIPRL in NSCLC was analyzed using The Cancer Genome Atlas (TCGA) dataset. Furthermore, Kaplan-Meier curve analysis was performed to evaluate the prognostic value of TIPRL in NSCLC, using the Kaplan-Meier Plotter and TCGA datasets. Loss-of-function assays were performed to determine the effects of TIPRL on cell migration and invasion. The results suggested that TIPRL was upregulated in NSCLC and positively associated with an advanced Tumor-Node-Metastasis stage. A higher expression level of TIPRL was associated with shorter overall and disease-free survival times in patients with NSCLC. To the best of our knowledge, the present study was the first to report that TIPRL acts as a metastasis promoter in NSCLC. Silencing of TIPRL suppressed A549 cell migration and invasion. Mechanistically, the present study indicated that TIPRL knockdown significantly promoted epithelial-cadherin expression, whereas it suppressed twist and vimentin expression in A549 cells. In conclusion, the present analysis suggested
\end{abstract}

Correspondence to: Professor Guofang Zhao, Department of Cardiothoracic Surgery, Hwa Mei Hospital, University of Chinese Academy of Sciences, 41 Northwestern Street, Ningbo, Zhejiang 315010, P.R. China

E-mail: guofangzhaonb@163.com

${ }^{*}$ Contributed equally

Key words: non-small cell lung cancer, TOR signaling pathway regulator, epithelial-to-mesenchymal transition, migration, invasion that TIPRL may serve as a biomarker for the prognosis of NSCLC and as a future target for its treatment.

\section{Introduction}

Non-small cell lung cancer (NSCLC) includes squamous cell carcinoma, adenocarcinoma and large-cell carcinoma and accounts for $>80 \%$ of all lung cancer cases globally (1). Despite various types of treatments developed for NSCLC in the past decades, the 5-year survival rate remains unsatisfactory at only $\sim 18 \%$ (2). Metastasis remains a leading cause of NSCLC-associated mortality (3). A number of metastasis regulators have been identified in NSCLC. For instance, WNT/TCF signaling was reported to regulate NSCLC metastasis through lymphoid enhancer binding factor 1 and HOXB9 (4). Jin et al (5) also indicated that LKB1 is involved in regulating lung cancer metastasis. However, the mechanisms underlying NSCLC metastasis remain to be further investigated (6).

TOR signaling pathway regulator (TIPRL), the mammalian ortholog of the yeast protein Tip41, is a type-2A phosphatase regulatory protein (7). Mechanistically, TIPRL may bind to protein phosphatase (PP)2A, PP4R2, PP4R3 and PP6 $(7,8)$. Previous studies have demonstrated that through the interaction between TIPRL and the PP4 complex, $\gamma$-H2AX becomes dephosphorylated to promote cell death (7), and that the interaction between TIPRL and PP2A leads to the activation of mTORC1-signaling activator (8). $\gamma-\mathrm{H} 2 \mathrm{AX}$ is a DNA damage response marker that may serve as a prognostic biomarker for cancer $(9,10)$. mTOR signaling has crucial roles in cancer progression and has been reported to be involved in regulating tumor growth (11), metastasis (12), autophagy (13), radioresistance (14) and chemoresistance (15). These studies indicated the important roles of TIPRL in human cancers. A recent study demonstrated that TIPRL was upregulated in hepatocellular carcinoma, while its knockdown induced cancer cell apoptosis (16). However, the molecular functions of TIPRL in NSCLC remain to be further investigated.

The present study focused on investigating the prognostic value and functional roles of TIPRL in NSCLC. The 
expression of TIPRL in NSCLC samples was assessed and the association between TIPRL expression and survival time was determined. Loss-of-function assays were performed to investigate the influence of TIPRL on NSCLC migration and invasion. The present results suggest that TIPRL may serve as a biomarker for the prognosis of patients with NSCLC and also as a therapeutic target.

\section{Materials and methods}

Datasets. The present study evaluated the expression levels of TIPRL in NSCLC samples using The Cancer Genome Atlas (TCGA) dataset (no. GSE27262), which was downloaded from the TCGA data portal (https://tcga-data.nci. nih.gov). The TCGA data subset for lung adenocarcinoma (LUAD) included 59 normal samples and 517 LUAD samples. Student's t-test was used to determine statistical significance between normal and LUAD samples. $\mathrm{P}<0.05$ was considered to indicate a statistically significant difference. The clinical information used in the study was downloaded from cBioPortal database (https://www.cbioportal.org/), which was uploaded as Table SI.

Cell culture. The NSCLC cell line A549 was purchased from the Cell Bank of the Type Culture Collection of the Chinese Academy of Sciences and cultured in RPMI-1640 medium (HyClone; GE Healthcare Life Sciences) supplemented with $10 \%$ fetal bovine serum (FBS; Gibco; Thermo Fisher Scientific, Inc.), penicillin $(100 \mathrm{U} / \mathrm{ml})$ and streptomycin $(100 \mathrm{U} / \mathrm{ml})$. The A549 cells were cultured at $37^{\circ} \mathrm{C}$ in a humidified atmosphere of $95 \%$ air and $5 \% \mathrm{CO}_{2}$.

Construction of TIPRL knockdown lentivirus. The short hairpin (sh)RNA sequence targeting TIPRL (5'-CCGGGTGCT GAAGAGTGGCAAGAAACTCGAGTTTCTTGCCACTCT TCAGCACTTTTT-3') was obtained from GeneChem, Inc. Recombinant lentiviral vectors were constructed according to previous studies (17). Concentrated lentiviruses were transfected at a multiplicity of infection of 40 in serum-free RPMI-1640 medium. The supernatant was replaced with complete culture medium (RPMI-1640 medium containing $10 \%$ FBS) after $24 \mathrm{~h}$. The transfection efficiency of shTIPRL was determined using reverse transcription-quantitative (RT-q)PCR and western blot analysis after $72 \mathrm{~h}$.

Cell proliferation and flow cytometric analysis. For cell proliferation assay, an MTT kit was used to detect the effect of TIPRL on cell proliferation. To dissolve the formazan crystals, $100 \mu$ dimethyl sulfoxide was added to each well and the absorbance at $570 \mathrm{~nm}$ was measured using a microplate reader. Data from three independent experiments were analyzed. For apoptosis assay, 100,000 cells/well A549 cell were seeded into 6 -well plates. A total of $48 \mathrm{~h}$ later, these cells were collected and washed twice using PBS. Then the apoptosis was detected using an Annexin V-APC Apoptosis Detection kit (eBioscience; Thermo Fisher Scientific, Inc.) by a flow cytometer (BD Biosciences; Becton, Dickinson and Company). Cell cycle distribution was analyzed as a typical DNA content histogram using BD CellQuest ${ }^{\mathrm{TM}}$ cell cycle analysis software (version 5.1; BD Biosciences).
Western blot analysis. Protein was extracted and western blot analysis was performed as described previously (18). The PVDF membranes were blocked with $5 \%$ skimmed milk at room temperature for $1 \mathrm{~h}$. The antibodies used in the experiments were as follows: Anti-TIPRL (1:1,000; cat. no. ab70795; Abcam), anti-GAPDH (1:1,000; cat. no. sc-32233; Santa Cruz Biotechnology, Inc.), anti-epithelial (E)-cadherin (1:1,000; cat. no. 3195; Cell Signaling Technology, Inc.), anti-vimentin (1:1,000; cat. no. 5741; Cell Signaling Technology, Inc.), anti-twist (1:1,000; cat. no. ab49254; Abcam) and anti-neural (N)-cadherin (1:1,000; cat. no. 13116; Cell Signaling Technology, Inc.). Goat anti-mouse IgG-horseradish peroxidase (1:4,000; cat. no. A4416; Sigma-Aldrich; Merck KGaA) and goat anti-rabbit IgG-horseradish peroxidase (1:4,000; cat. no. A0545; Sigma-Aldrich; Merck KGaA) secondary antibodies were used. ECL chemiluminescence reagent (Santa Cruz Biotechnology, Inc.) was used to visualize the bands. The grey values of the protein bands were quantified with ImageJ software (version 1.8; National Institutes of Health).

$R T$ - $q P C R$. PCR analysis was performed as described previously (18). Total RNA was extracted from cells using TRIzol ${ }^{\circledR}$ reagent (Invitrogen; Thermo Fisher Scientific, Inc.) and complementary (c)DNA was synthesized using the RevertAid First Strand cDNA Synthesis kit (Promega Corp.). The reverse transcription conditions were: $25^{\circ} \mathrm{C}$ for $10 \mathrm{~min}, 37^{\circ} \mathrm{C}$ for $120 \mathrm{~min}$ and $85^{\circ} \mathrm{C}$ for $5 \mathrm{~min}$. The miScriptSYBR GreenPCR kit (Qiagen, Inc.) was used to perform qPCR. The PCR amplification was performed at $95^{\circ} \mathrm{C}$ for $30 \mathrm{sec}$, followed by 40 cycles of denaturation at $95^{\circ} \mathrm{C}$ for $5 \mathrm{sec}$ and annealing/extension at $60^{\circ} \mathrm{C}$ for $30 \mathrm{sec}$ using an ABI 7300 Thermocycler (Applied Biosystems; Thermo Fisher Scientific, Inc.). The qPCR primers used in the present study were TIPRL forward, 5'-GGCGTC CAAGACCCACATC-3' and reverse, 5'-ACAGGCCACTTT AAGCATTCC-3'; and GAPDH forward, 5'-TGACTTCAA CAGCGACACCCA-3', and reverse, 5'-CACCCTGTTGCT GTAGCCAAA-3'.

Wound healing assay. A549 cells were seeded into a 6-well plate until the cells were confluent and cultured in serum-free medium for $24 \mathrm{~h}$ to obtain a monolayer. A scratch was made using an Oris ${ }^{\mathrm{TM}}$ plate. The cells were rinsed with PBS and incubated with fresh serum-free medium at $37^{\circ} \mathrm{C}$. Images were captured at 0,24 and $48 \mathrm{~h}$ using an inverted light microscope (TS100; Nikon Corporation) and analyzed using ImageJ. Each assay was performed in triplicate.

Transwell assay. Transwell assays were performed using Transwell inserts with an $8-\mu \mathrm{m}$ pore size. The cell invasion assay was performed in Transwell plates pre-coated with Matrigel Basement Membrane Matrix at $37^{\circ} \mathrm{C}$ for $1 \mathrm{~h}$ (coating concentration, $1 \mathrm{mg} / \mathrm{ml}$; BD Biosciences). After transfection, A549 cells were seeded into the upper well at $5 \times 10^{4}$ cells/well in RPMI-1640 medium containing 1\% (fetal bovine serum) FBS. Medium with $10 \%$ FBS was added to the bottom wells of the system. After 3 days of incubation, the cells on the upper and lower sides of the Transwell membrane were fixed with $4 \%$ paraformaldehyde at $37^{\circ} \mathrm{C}$ for $30 \mathrm{~min}$ and stained with $0.5 \%$ crystal violet for $5 \mathrm{~min}$ at room temperature. The cells on the upper side, which had not transgressed through 

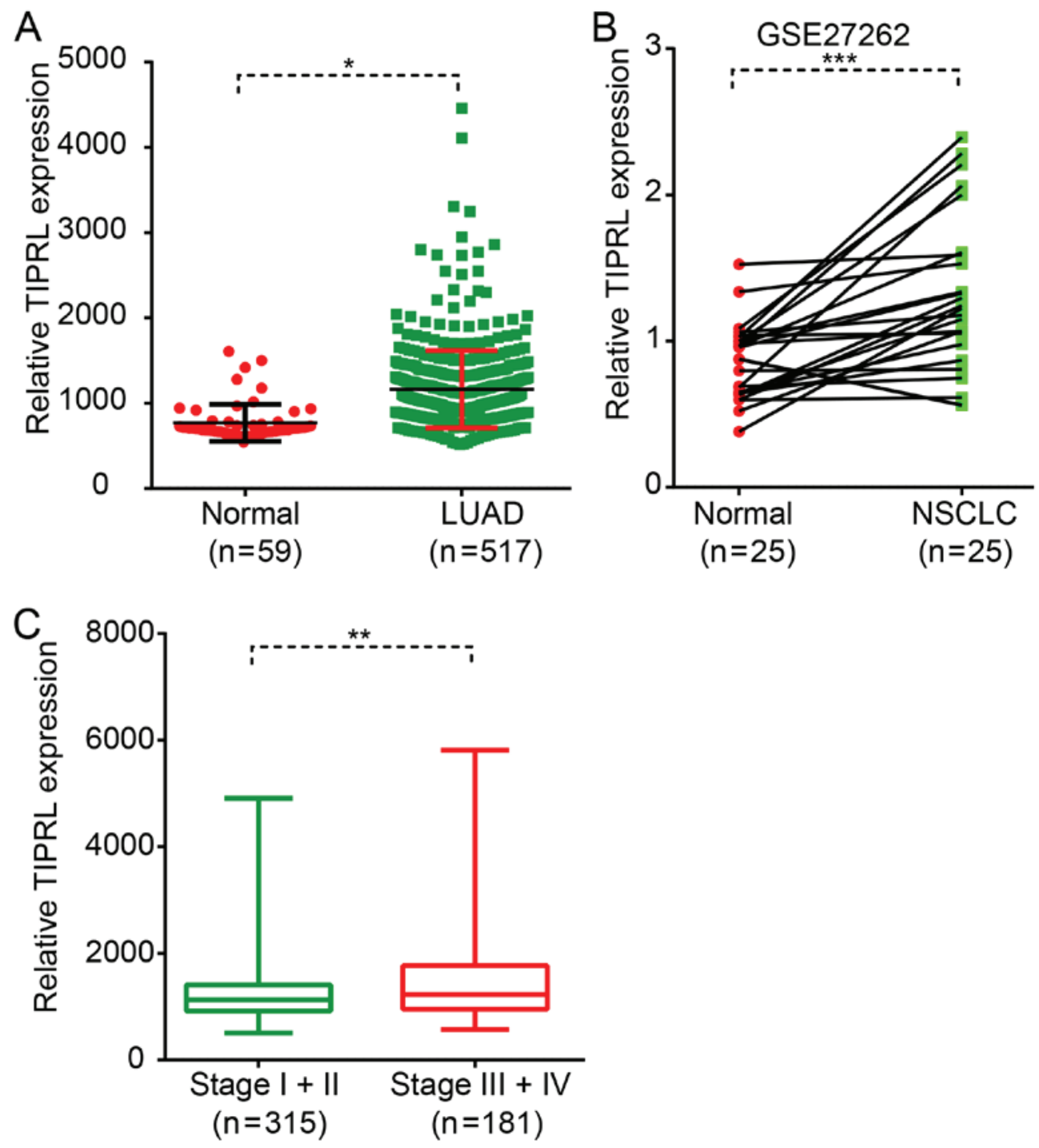

Figure 1. TIPRL is upregulated in NSCLC. (A) TIPRL was upregulated in LUAD samples compared with normal samples. (B) TIPRL was upregulated in NSCLC samples compared with normal samples by analyzing GSE27262. (C) TIPRL was upregulated in Stage III and IV LUAD samples compared with Stage I and II LUAD samples. ${ }^{*} \mathrm{P}<0.05,{ }^{* *} \mathrm{P}<0.01$ and ${ }^{* * * *} \mathrm{P}<0.001$. LUAD, lung adenocarcinoma; TIPRL, TOR signaling pathway regulator; NSCLC, non-small cell lung cancer.

the membrane, were gently removed with a cotton swab and the numbers of invasive cells in 5 random fields of view were counted under a light microscope (magnification, x100; Olympus Corporation).

Statistical analysis. All experiments were performed as 3 independent biological replicates and data are presented as the mean \pm standard deviation. All statistical analyses were performed using SPSS software version 16.0 (SPSS, Inc.). Student's t-test was used to determine statistical significance between two groups. For multiple groups, one-way analysis of variance followed by the Newman-Keuls post hoc test was used. $\mathrm{P}<0.05$ was considered to indicate a statistically significant difference.

\section{Results}

TIPRL is upregulated in NSCLC. The expression pattern of TIPRL in NSCLC has remained elusive. The present study evaluated the expression levels of TIPRL in NSCLC and normal lung samples by analyzing a public dataset, LUAD, which was downloaded from TCGA database. As presented in Fig. 1, TIPRL was identified to be significantly overexpressed in LUAD samples compared with in the matched normal tissues $(\mathrm{P}<0.05$; Fig. 1A). In order to further validate the present findings, an independent dataset, GSE27262 was analyzed, which was based on the microarray technology. The results also showed that TIPRL was significantly overexpressed in NSCLC samples compared with the normal tissues $(\mathrm{P}<0.001$; Fig. 1B). Of note, higher TIPRL expression was associated with the advanced stage of NSCLC. The present results demonstrated that TIPRL was significantly upregulated in stage-3 and stage-4 LUAD samples compared with that in stage-1 and stage-2 LUAD samples $(\mathrm{P}<0.01$; Fig. 1C).

TIPRL is correlated with a shorter survival time in NSCLC. Kaplan-Meier curve analysis was then performed to evaluate 

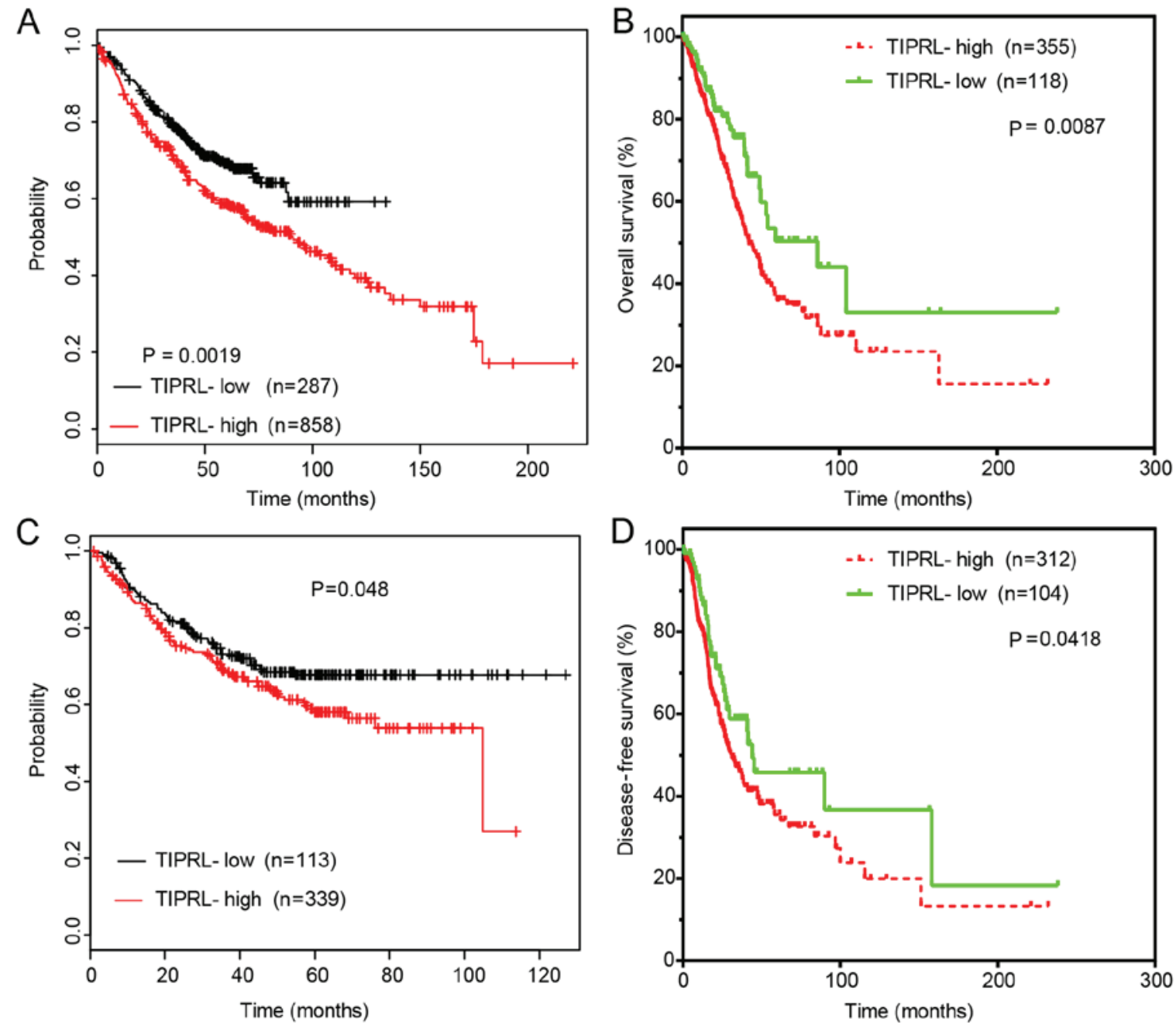

Figure 2. Higher TIPRL expression is associated with shorter overall survival and disease-free time in NSCLC. (A and B) Higher TIPRL expression was associated with shorter overall survival time in NSCLC by analyzing (A) TCGA and Kaplan-Meier (B) Plotter datasets. (C and D) Higher TIPRL expression was associated with shorter disease-free survival time in NSCLC by analyzing (C) TCGA and (D) Kaplan-Meier Plotter datasets. LUAD, lung adenocarcinoma; TIPRL, TOR signaling pathway regulator; NSCLC, non-small cell lung cancer; TCGA, The Cancer Genome Atlas.

the prognostic value of TIPRL in NSCLC. The upper quartile of TIPRL expression in LUAD samples was selected as the cutoff. First, the Kaplan-Meier Plotter dataset was analyzed. The results demonstrated that high TIPRL expression in lung cancer samples was associated with shorter overall survival (OS) (Fig. 2A) and disease-free survival (DFS) time (Fig. 2C). Analysis of TCGA datasets indicated that high TIPRL expression levels in LUAD samples were associated with reduced OS (Fig. 2B) and DFS time (Fig. 2D). These results suggested that dysregulation of TIPRL may indicate the prognosis of NSCLC.

Silencing of TIPRL inhibits NSCLC cell proliferation. An shRNA was designed to knock down endogenous expression of TIPRL in NSCLC. As presented in Fig. 3, the mRNA (Fig. 3A) and protein (Fig. 5A) expression of TIPRL was significantly decreased in A549 cells ( $\mathrm{P}<0.01)$. By using an MTT assay, it was found that silencing of TIPRL significantly suppressed A549 cell proliferation compared with the control group after 4 days $(\mathrm{P}<0.001$; Fig. 3B). Flow cytometric analysis showed that silencing of TIPRL induced A549 cell apoptosis by 2-fold compared with the control group (Fig. 3C and D). These results suggested that TIPRL played an important role in regulating cancer proliferation.

Silencing of TIPRL inhibits NSCLC cell migration and invasion. A wound healing assay was first performed to detect the influence of TIPRL on cell metastasis. The repair rate in the TIPRL knockdown group was significantly suppressed in comparison with that in the control group $(\mathrm{P}<0.05)$. The results demonstrated that 11 and $23 \%$ of the wound area in the TIPRL knockdown group was repaired by migrating cells after 24 and $48 \mathrm{~h}$, respectively. However, 30 and $45 \%$ of the wounded area in the control groups was repaired under the same incubation conditions (Fig. 3E and F).

Next, Transwell assays were performed. It was demonstrated that the numbers of migrating A549 cells were significantly decreased by $80 \%$ in the TIPRL knockdown group compared with those in the control group ( $\mathrm{P}<0.001$; Fig. 4A and B). In an invasion assay using Matrigel, it was observed that silencing of TIPRL significantly inhibited the invasion ability of A549 cells by $70 \%$ compared with that in the control groups $(\mathrm{P}<0.001$; Fig. 4C and D). These results indicated that TIPRL acts as a negative regulator of metastasis in NSCLC. 
A

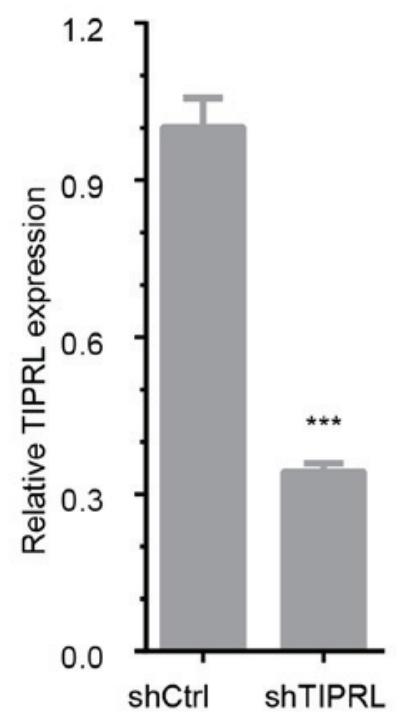

C

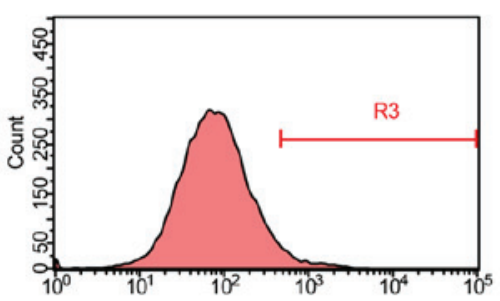

Red-R fluorescence (RED-R-HLog) Red-R fluorescence (RED-R-HLog)

B

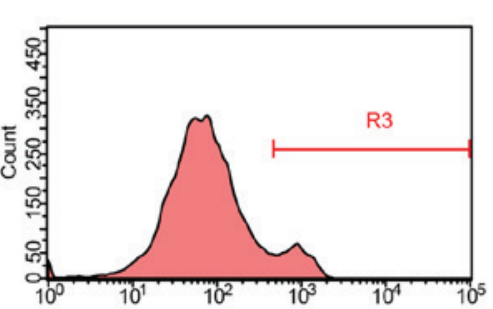

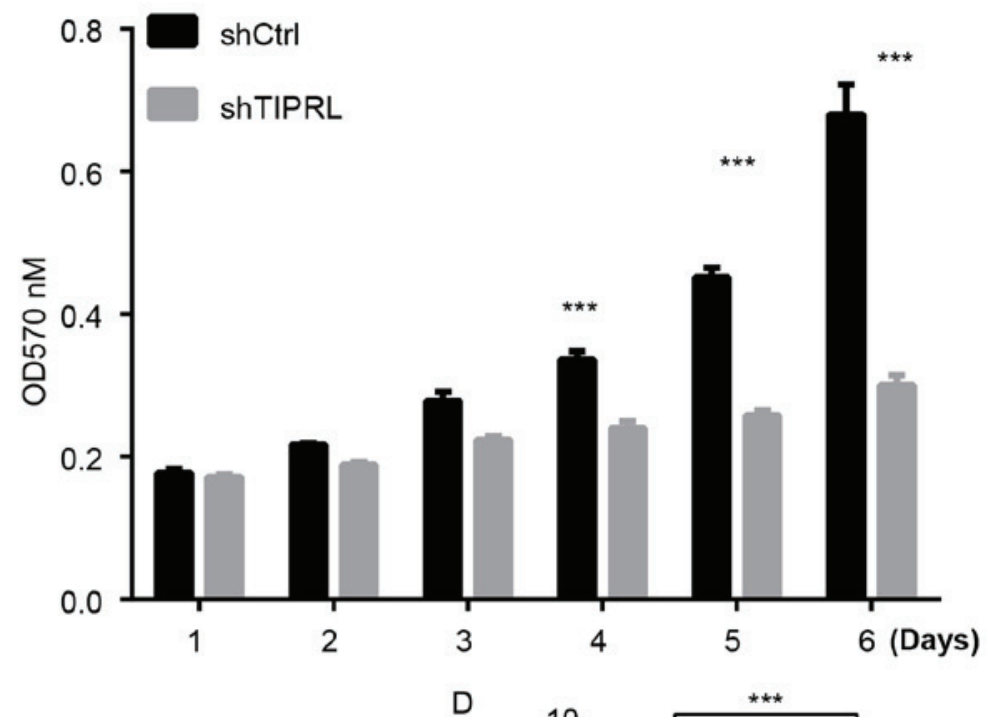

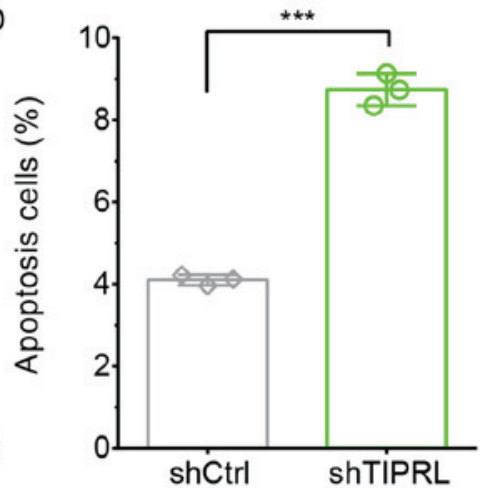

$\mathrm{F}$

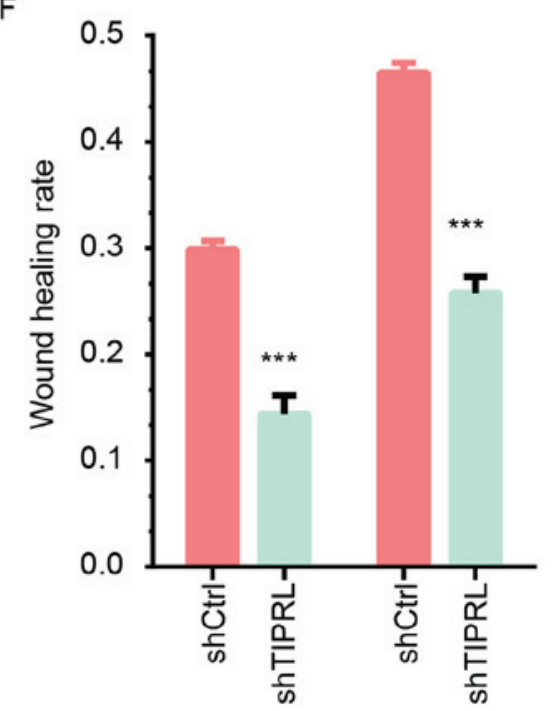

Figure 3. Knockdown of TIPRL suppresses NSCLC migration using a transwell assay. (A) TIPRL special shRNA suppressed its endogenous expression by using reverse transcription-PCR (B) TIPRL knockdown significantly inhibits cell proliferation in NSCLC cells by performing an MTT assay. (C) TIPRL knockdown significantly induced cell apoptosis in NSCLC cells and (D) statistical analysis of the results. (E) TIPRL knockdown significantly inhibits cell migration in NSCLC cells by performing a wound healing assay. Magnification, x100. (F) Quantitative analysis showed TIPRL knockdown significantly inhibits cell migration in NSCLC cells. Data are presented as the mean \pm standard deviation $(n=3){ }^{* * *} \mathrm{P}<0.001$. TIPRL, TOR signaling pathway regulator; NSCLC, non-small cell lung cancer; TCGA, The Cancer Genome Atlas; sh, short hairpin; OD, optical density; Ctrl, control.

Knockdown of TIPRL inhibits epithelial-to-mesenchymal transition (EMT) in NSCLC. In order to explore whether TIPRL promoted metastasis through regulating EMT, the protein levels of E-cadherin, twist, vimentin and $\mathrm{N}$-cadherin were detected after TIPRL knockdown in NSCLC. Western blot analysis indicated that E-cadherin was significantly induced $(\mathrm{P}<0.05)$, whereas the mesenchymal markers twist, vimentin and E-cadherin were significantly reduced in 

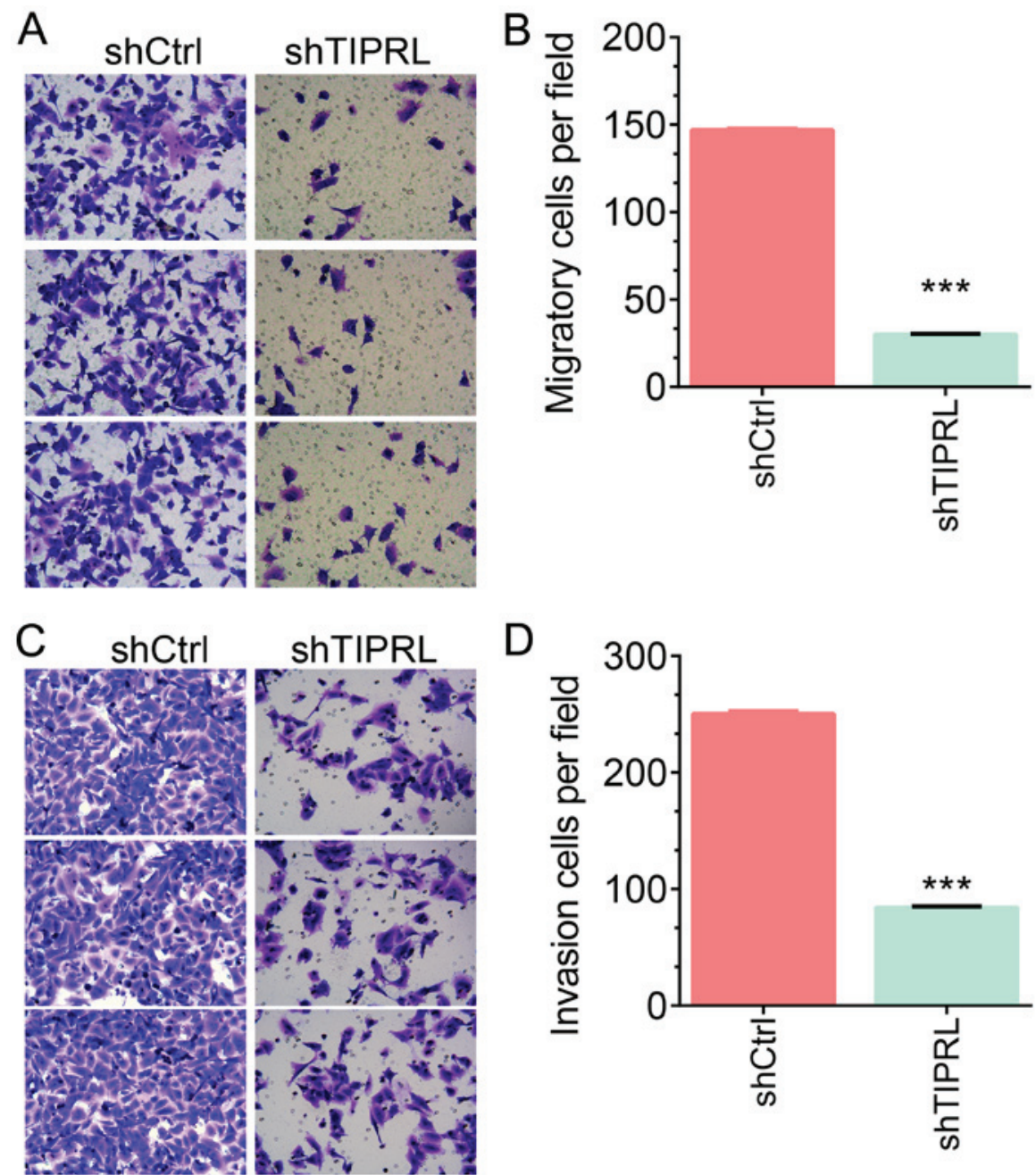

Figure 4. Knockdown of TIPRL suppressed NSCLC migration and invasion using transwell assay. TIPRL knockdown significantly inhibits cell (A) migration and (C) invasion in NSCLC cells by performing transwell assay. Quantitative analysis showed TIPRL knockdown significantly inhibits cell (B) migration and (D) invasion in NSCLC cells. Data are presented as the mean \pm standard deviation $(n=3)^{* * * *} \mathrm{P}<0.001$. TIPRL, TOR signaling pathway regulator; NSCLC, non-small cell lung cancer; TCGA, The Cancer Genome Atlas; sh, short hairpin; Ctrl, control.

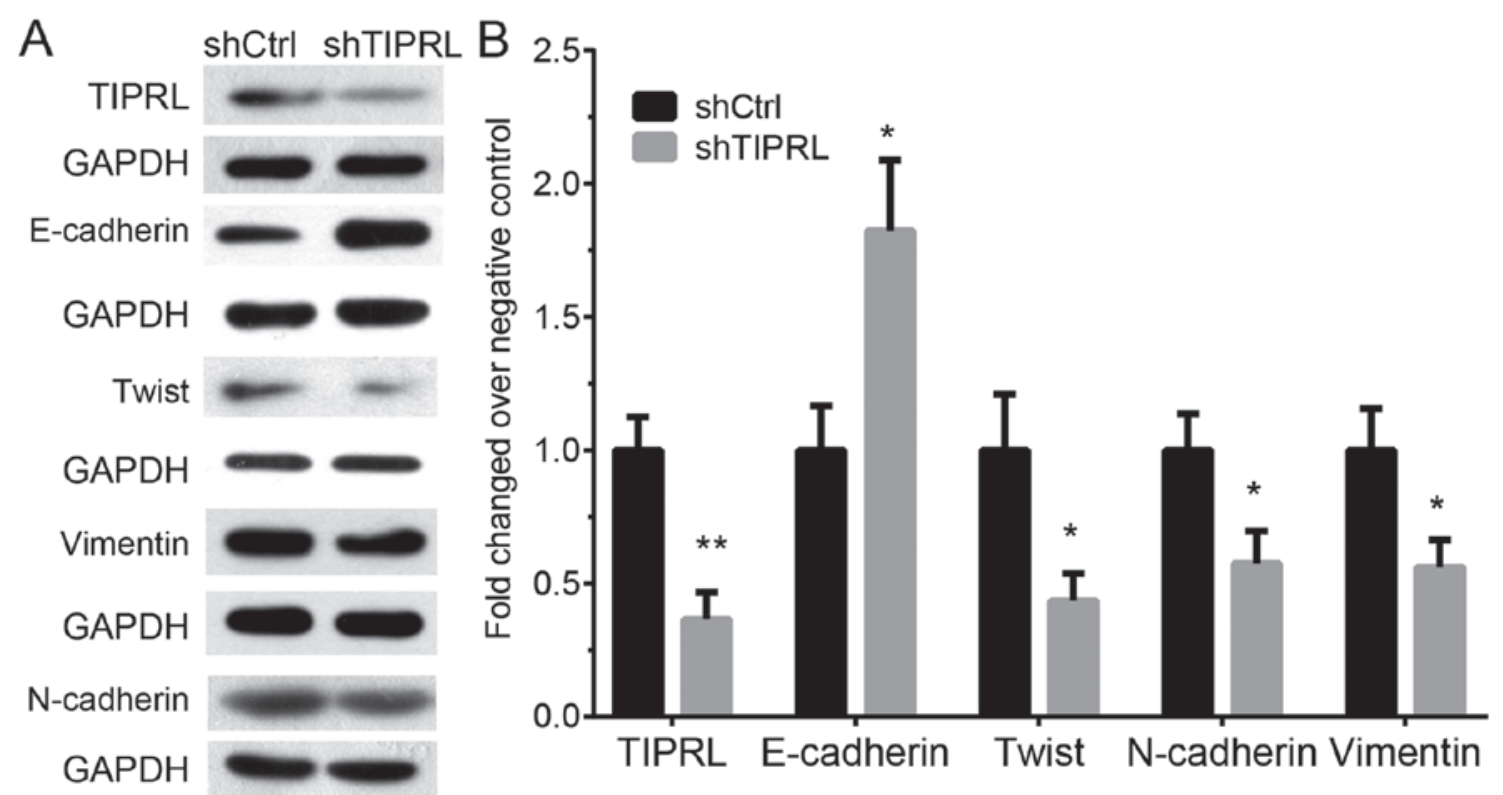

Figure 5. Knockdown of TIPRL suppressed epithelial mesenchymal transition. (A) TIPRL knockdown treatment significantly promoted E-cadherin expression, whereas suppressed twist and vimentin expression in A549 cells. (B) Relative grey values of each band (normalized to GAPDH). ${ }^{*} \mathrm{P}<0.05$ and ${ }^{* *} \mathrm{P}<0.01$ vs. control group. TIPRL, TOR signaling pathway regulator; E, epithelial; sh, short hairpin; Ctrl, control; N, neural. 
TIPRL-knockdown A549 cells compared with those in the control groups $(\mathrm{P}<0.05$; Fig. 5). These results suggested that knockdown of TIPRL inhibited EMT in NSCLC.

\section{Discussion}

Previous studies had demonstrated that TIPRL played an important role in regulating cancer progression $(8,16)$. TIPRL acts as an activator of mTORC1 signaling. TIPRL was overexpressed and served crucial roles in human liver cancer. TIPRL was reported to be involved in regulating cell apoptosis and cell death through binding to PP2Ac and PP4. Yoon et al (16) indicated that knockdown of TIPRL in hepatocellular carcinoma induced tumor-cell apoptosis. Rosales et al (7) reported that TIPRL induced cell death in response to genotoxic stress. It has also been shown that knockdown of TIPRL enhances the apoptosis of cisplatin-treated cells in lung cancer (19). TIPRL associates with MKK7 and activates JNK thereby regulating the apoptotic pathway via caspase 3 and 9 release (20). Moreover, TIPRL acts as an activator of mTORC1 signaling, which is a key regulator of metabolic pathways and plays a crucial role in promoting cancer growth (8). The present study also showed that knockdown of TIPRL suppressed NSCLC cell proliferation and induced cell apoptosis. To the best of our knowledge, the present study was the first to report that TIPRL acts as a metastasis promoter in NSCLC. Knockdown of TIPRL suppressed NSCLC migration and invasion through regulating EMT processes. The present study provides insight into a potential regulatory mechanism involved in NSCLC.

Metastasis is a leading cause of NSCLC-associated mortality. A series of NSCLC metastasis regulators were identified in previous studies. For instance, transmembrane protein 106B was reported to drive lung cancer metastasis through promoting the synthesis of enlarged vesicular lysosomes (21). A previous study indicated that GATAD2B promoted KRAS-driven lung cancer metastasis (22). Understanding the detailed mechanisms underlying NSCLC metastasis may provide novel information to develop more effective therapeutic strategies. EMT has crucial roles in NSCLC metastasis (23). During EMT, the epithelioid cell markers, including E-cadherin, are downregulated, while interstitial-cell markers, including $\mathrm{N}$-cadherin and vimentin, are upregulated. Transforming growth factor- $\beta$ signaling and $\mathrm{Wnt} / \beta$-catenin have key roles in the regulation of EMT processes. In NSCLC, several genes, including kinesin family member C1 (24), APE1 $(25,26)$ and F-box and WD repeat domain containing 7 (27), were reported to participate in the regulation of EMT. The present study demonstrated that knockdown of TIPRL in A549 cells promoted E-cadherin expression, whereas suppressed twist and vimentin expression was observed. Taken together, the present study demonstrated that TIPRL is a promoter of EMT.

In the past decades, a number of biomarkers, including carcinoembryonic antigen, cytokeratin-19 fragment and CYFRA 21-1, were identified in lung cancer (28). However, the sensitivity and specificity of these markers remain unsatisfactory. To date, the prognostic value of TIPRL in NSCLC has remained elusive. The present study indicated that TIPRL was overexpressed in NSLC tissues. A higher expression of TIPRL was associated with higher lymphatic metastasis and
Tumor-Node-Metastasis stage. Furthermore, overexpression of TIPRL in NSCLC tissues was associated with shorter OS and DFS time in NSCLC patients. These results strongly suggested that TIPRL may serve as a novel biomarker for NSCLC.

In conclusion, the present study suggested that TIPRL was upregulated and associated with shorter survival time in NSCLC. Furthermore, silencing of TIPRL inhibited NSCLC migration and invasion through regulating EMT. The present study presents a potential novel biomarker for NSCLC, which may be utilized as a therapeutic target and/or prognostic factor.

\section{Acknowledgements}

Not applicable.

\section{Funding}

No funding was received.

\section{Availability of data and materials}

The datasets used and/or analyzed during the current study are available from the corresponding author on reasonable request.

\section{Authors' contributions}

$\mathrm{XX}, \mathrm{HZ}$ and GZ designed the experiments. XX, HZ, MY, EZ, and YZ performed the experiments. XX, JN, RL, ZY and TH collected and analyzed the data. XX and GZ drafted the manuscript. All authors approved the manuscript for publication.

\section{Ethics approval and consent to participate}

Not applicable.

\section{Patient consent for publication}

Not applicable.

\section{Competing interests}

The authors declare that they have no competing interests.

\section{References}

1. Zhao B, Han H, Chen J, Zhang Z, Li S, Fang F, Zheng Q, Ma Y, Zhang J, Wu N and Yang Y: MicroRNA let-7c inhibits migration and invasion of human non-small cell lung cancer by targeting ITGB3 and MAP4K3. Cancer Lett 342: 43-51, 2014.

2. Gao L, Qiu H, Liu J, Ma Y, Feng J, Qian L, Zhang J, Liu Y and Bian T: KLF15 promotes the proliferation and metastasis of lung adenocarcinoma cells and has potential as a cancer prognostic marker. Oncotarget 8: 109952-109961, 2017.

3. Waqar SN, Samson PP, Robinson CG, Bradley J, Devarakonda S, Du L, Govindan R, Gao F, Puri V and Morgensztern D: Non-small-cell lung cancer with brain metastasis at presentation. Clin Lung Cancer 19: e373-e379, 2018.

4. Nguyen DX, Chiang AC, Zhang XH, Kim JY, Kris MG, Ladanyi M, Gerald WL and Massagué J: WNT/TCF signaling through LEF1 and HOXB9 mediates lung adenocarcinoma metastasis. Cell 138: 51-62, 2009.

5. Jin L, Chun J, Pan C, Kumar A, Zhang G, Ha Y, Li D, Alesi GN, Kang Y, Zhou L, et al: The PLAG1-GDH1 axis promotes anoikis resistance and tumor metastasis through CamKK2-AMPK signaling in LKB1-deficient lung cancer. Mol Cell 69: 87-99 e7, 2018. 
6. Ji H, Ramsey MR, Hayes DN, Fan C, McNamara K, Kozlowski P, Torrice C, Wu MC, Shimamura T, Perera SA, et al: LKB1 modulates lung cancer differentiation and metastasis. Nature 448: 807-810, 2007

7. Rosales KR, Reid MA, Yang Y, Tran TQ, Wang WI, Lowman X, Pan $\mathrm{M}$ and Kong M: TIPRL inhibits protein phosphatase 4 activity and promotes $\mathrm{H} 2 \mathrm{AX}$ phosphorylation in the DNA damage response. PLoS One 10: e0145938, 2015.

8. Nakashima A, Tanimura-Ito K, Oshiro N, Eguchi S, Miyamoto T, Momonami A, Kamada S, Yonezawa K and Kikkawa U: A positive role of mammalian Tip41-like protein, TIPRL, in the amino-acid dependent mTORC1-signaling pathway through interaction with PP2A. FEBS Lett 587: 2924-2929, 2013.

9. Chatzimichail E, Matthaios D, Bouros D, Karakitsos P, Romanidis K, Kakolyris S, Papashinopoulos G and Rigas A: $\gamma$-H2AX: A novel prognostic marker in a prognosis prediction model of patients with early operable non-small cell lung cancer. Int J Genomics 2014: 160236, 2014.

10. Matthaios D, Hountis P, Karakitsos P, Bouros D and Kakolyris S: $\mathrm{H} 2 \mathrm{AX}$ a promising biomarker for lung cancer: A review. Cancer Invest 31: 582-599, 2013.

11. Roy D, Sin SH, Lucas A, Venkataramanan R, Wang L, Eason A, Chavakula V, Hilton IB, Tamburro KM, Damania B and Dittmer DP: mTOR inhibitors block Kaposi sarcoma growth by inhibiting essential autocrine growth factors and tumor angiogenesis. Cancer Res 73: 2235-2246, 2013.

12. Zhou H and Huang S: Role of mTOR signaling in tumor cell motility, invasion and metastasis. Curr Protein Pept Sci 12: 30-42, 2011.

13. Paquette M, El-Houjeiri L and Pause A: mTOR pathways in cancer and autophagy. Cancers (Basel) 10: pii: E18, 2018.

14. Chang L, Graham PH, Ni J, Hao J, Bucci J, Cozzi PJ and Li Y: Targeting PI3K/Akt/mTOR signaling pathway in the treatment of prostate cancer radioresistance. Crit Rev Oncol Hematol 96: $507-517,2015$.

15. Tian L, Zhao Z, Xie L and Zhu J: MiR-361-5p suppresses chemoresistance of gastric cancer cells by targeting FOXM1 via the PI3K/Akt/mTOR pathway. Oncotarget 9: 4886-4896, 2017.

16. Yoon JY, Lee JJ, Gu S, Jung ME, Cho HS, Lim JH, Jun SY, Ahn JH, Min JS, Choi MH, et al: Novel indazole-based small compounds enhance TRAIL-induced apoptosis by inhibiting the MKK7-TIPRL interaction in hepatocellular carcinoma. Oncotarget 8: 112610-112622, 2017.

17. Cui F, Hu J, Ning S, Tan J and Tang H: Overexpression of MCM10 promotes cell proliferation and predicts poor prognosis in prostate cancer. Prostate 78: 1299-1310, 2018.
18. Kroiss A, Vincent S, Decaussin-Petrucci M, Meugnier E, Viallet J, Ruffion A, Chalmel F, Samarut J and Allioli N: Androgen-regulated microRNA-135a decreases prostate cancer cell migration and invasion through downregulating ROCK1 and ROCK2. Oncogene 34: 2846-2855, 2015.

19. Park JY and Juhnn YS: cAMP signaling increases histone deacetylase 8 expression via the Epac2-Rap1A-Akt pathway in H1299 lung cancer cells. Exp Mol Med 49: e297, 2017.

20. Song IS, Jun SY, Na HJ, Kim HT, Jung SY, Ha GH, Park YH, Long LZ, Yu DY, Kim JM, et al: Inhibition of MKK7-JNK by the TOR signaling pathway regulator-like protein contributes to resistance of HCC cells to TRAIL-induced apoptosis. Gastroenterology 143: 1341-1351, 2012.

21. Kundu ST, Grzeskowiak CL, Fradette JJ, Gibson LA, Rodriguez LB, Creighton CJ, Scott KL and Gibbons DL: TMEM106B drives lung cancer metastasis by inducing TFEB-dependent lysosome synthesis and secretion of cathepsins. Nat Commun 9: 2731, 2018.

22. Grzeskowiak CL, Kundu ST, Mo X, Ivanov AA, Zagorodna O, Lu H, Chapple RH, Tsang YH, Moreno D, Mosqueda M, et al: In vivo screening identifies GATAD2B as a metastasis driver in KRAS-driven lung cancer. Nat Commun 9: 2732, 2018.

23. Brody H: Lung cancer. Nature 513: S1, 2014.

24. Takeyama Y, Sato M, Horio M, Hase T, Yoshida K, Yokoyama T, Nakashima H, Hashimoto N, Sekido Y, Gazdar AF, et al: Knockdown of ZEB1, a master epithelial-to-mesenchymal transition (EMT) gene, suppresses anchorage-independent cell growth of lung cancer cells. Cancer Lett 296: 216-224, 2010.

25. Han J, Wang F, Lan Y, Wang J, Nie C, Liang Y, Song R, Zheng T, Pan S, Pei T, et al: KIFC1 regulated by miR-532-3p promotes epithelial-to-mesenchymal transition and metastasis of hepatocellular carcinoma via gankyrin/AKT signaling. Oncogene 38: 406-420, 2019

26. Yang X, Peng Y, Jiang X, Lu X, Duan W, Zhang S, Dai N, Shan J, Feng Y, Li X, et al: The regulatory role of APE1 in epithelial-to-mesenchymal transition and in determining EGFR-TKI responsiveness in non-small-cell lung cancer. Cancer Med 7: 4406-4419, 2018.

27. Wei X,LiQ, Li Y, Duan W, Huang C, Zheng X, Sun L, Luo J, Wang D, Zhang S, et al: Prediction of survival prognosis of non-small cell lung cancer by APE1 through regulation of Epithelial-Mesenchymal Transition. Oncotarget 7: 28523-28539, 2016.

28. Zhang Y, Zhang X, Ye M, Jing P, Xiong J, Han Z, Kong J, Li M, Lai X, Chang N, et al: FBW7 loss promotes epithelial-tomesenchymal transition in non-small cell lung cancer through the stabilization of Snail protein. Cancer Lett 419: 75-83, 2018. 